\title{
The planar Multipole Resonance Probe: a functional analytic approach
}

\author{
M. Friedrichs ${ }^{*}$ (D) and J. Oberrath
}

\author{
${ }^{*}$ Correspondence: \\ michael.friedrichs@leuphana.de \\ Institute of Product- and Process \\ Innovation, Department of \\ Economic Sciences, Leuphana \\ University Lüneburg, D-21339 \\ Lüneburg, Germany
}

\begin{abstract}
Active Plasma Resonance Spectroscopy (APRS) is a well known diagnostic method, where a radio frequency probe is immersed into a plasma and excites plasma oscillations. The response of the plasma is recorded as frequency dependent spectrum, in which resonance peaks occur. By means of a mathematical model plasma parameters like the electron density or the electron temperature can be determined from the detected resonances.

The majority of all APRS probes have in common, that they are immersed into the plasma and perturb the plasma due to the physical presence of the probe. Thus, they are invasive and can at least influence the homogeneity of the plasma. To overcome this problem, the planar Multipole Resonance Probe (pMRP) was invented, which can be integrated into the chamber wall of a plasma reactor.

Within this paper, the first analytic model of the PMRP is presented, which is based on a cold plasma description of the electrons. The general admittance of the probe-plasma system is derived by means of functional analytic methods and a complete orthonormal set of basis functions. Explicit spectra for an approximated admittance including a convergence study are shown. The determined resonance frequencies are in good agreement with former simulation results.

Keywords: Active Plasma Resonance Spectroscopy, Multipole Resonance Probe, non-invasive Plasma Process Monitoring, Plasma Diagnostic, Functional analysis, planar Multipole Resonance Probe
\end{abstract}

\section{Introduction}

A plasma occupies the natural ability to resonate near the electron plasma frequency $\omega_{\text {pe }}$. This ability is the essential requirement for active plasma resonance spectroscopy (APRS). Coupling a radio frequency (rf) signal in the $\mathrm{GHz}$ range into the plasma via an electrical probe the frequency dependent system response can be recorded to detect resonances. By means of a mathematical model to describe these resonance phenomena plasma parameters like electron density can be calculated.

Many different designs of APRS probes are invented. An overview and a classification of them are presented in reference [1]. One class of APRS probes excites electrostatic resonances [2-9], which occur below $\omega_{\text {pe }}$ and can be described by a model of the probeplasma system in an electrostatic approximation. Many approaches to understand these resonance phenomena have been reported [10-25]. They have in common, that their

(c) The Author(s). 2018 licensee Springer on behalf of EPJ. This is an Open Access article distributed under the terms of the Creative Commons Attribution License (http://creativecommons.org/licenses/by/4.0), which permits unrestricted use, distribution, and reproduction in any medium, provided the original work is properly credited. 
models are based on a fluid dynamical description and they focus only on a specific design of a certain probe.

However, the whole class of these probes can also be described generally. Applying functional analytic (Hilbert space) methods, a general and geometry independent solution of the system response can be derived [26]. This response is identified as the electrical admittance of the probe-plasma system. Based on this solution one can prove, that the Multipole Resonance Probe (MRP) has the optimal design [26, 27].

Apart from this fact, it is not suited for many industrial applications, because it is immersed into the plasma and thus invasive due to its physical presence. In industry non-invasive diagnostic methods are preferred, which have ideally no influence on the homogeneity of the plasma. To minimize the invasive behavior of the MRP a planar version, the planar Multipole Resonance Probe (pMRP), was invented [28]. First measurements for two different plasma densities show a clear shift of the resonance frequency to a higher value, which was also shown in CST-simulations. A more detailed investigation is presented in ref. [29], where the influence of the electrode radius was analyzed. The simulation results show, that a certain radius has to be chosen to cover a specific frequency range. Also a variation of the elastic collision frequency in the range of 300 to $600 \mathrm{MHz}$ was investigated and compared to the behavior of the MRP. Measurements with the pMRP of a radius of $4 \mathrm{~mm}$ for different plasma input powers between $200 \mathrm{~W}$ and $500 \mathrm{~W}$ in a double inductively coupled plasma in argon were compared to the simulation results and show a comparable behavior.

However, to determine plasma parameters from the measured resonance peaks, a mathematical model is needed. In this manuscript the general description of APRS in an electrostatic approximation will be applied to the geometry of the pMRP and an analytic solution for the admittance of the probe-plasma system will be presented. To determine specific spectra, the analytic solution has to be approximated, which requires a convergence study for different parameters. The final converged spectra lead to a proportional relation between the resonance and the plasma frequency, which can be used as a simple formula to determine the electron density from a measured resonance. The definition of such a simple relation is the main goal of this manuscript.

\section{Consequences of symmetry in MRP and pMRP design}

In reference [26] a geometry independent APRS probe, which excites electrostatic resonance modes, was analyzed. In the general APRS model a matrix sheath with complete electron depletion and a sharp sheath edge is assumed. One of the main results was, that the physical interpretation of the probe-plasma interaction can be represented by a lumped element circuit model. An example for a two electrode system was presented. The generalized structure of this circuit can be identified as a triangle and is depicted in Fig. 1 (left). It shows a coupling of each electrode to ground and a coupling between the electrodes itself. In case of any two electrode probe-plasma system, which fulfills a geometric symmetry between the electrodes, leads to equal impedances $Z_{2}$ in the branches between the electrodes and ground.

Each triangle circuit can be transformed equivalently into a star circuit (see Fig. 1, center). Thus, the impedances $Z_{12}$ conducted to the electrodes have to be equal, due to the geometric symmetry. Applying anti-symmetric voltages (with a 180 degree phase shift) at the two electrodes, the current from the upper electrode to ground equals the current 

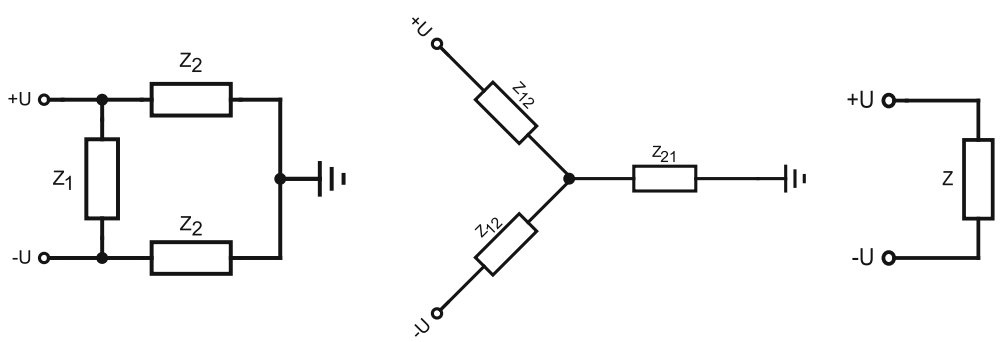

Fig. 1 General lumped element circuit model of a two electrode probe-plasma system in geometric symmetry (left), equivalent star circuit of this system (center), and simplified circuit without coupling to ground due to anti-symmetric voltages applied to the electrodes (right)

from ground to the lower electrode, but with opposite directions. Due to that, these currents vanish and a virtual ground is present in the center of the star circuit, which allows to neglect the coupling to ground. The simplified lumped element circuit model of the probe-plasma interaction is depicted in Fig. 1 (right). A $R L C$ series circuit parallel to a vacuum capacitance $C_{\mathrm{vac}}$ is the simplest lumped element circuit model for $Z$ as shown in [26]. $C$ represents the dielectric and sheath behavior, $L$ the inertia of the electrons, and $R$ damping due to elastic collisions of the electrons with the neutral background. $C_{\mathrm{vac}}$ represents the coupling of the electrodes, which is also present without plasma. Thus, the probe measures the series resonance of the plasma and the sheath.

The required symmetries are fulfilled by both, the MRP and also the pMRP. A schematic of the pMRP is shown in Fig. 2. It consists of two half-disc electrodes, which are conducted to a tapered-balun. This balun ensures the anti-symmetric signal at the electrodes, which is generated as a non-symmetric signal by a network analyzer and transferred to the balun via a coaxial cable. To avoid a direct contact of the electrodes with the plasma, they are covered by a dielectric disc, which has a larger radius as the electrodes. The whole probe, including the dielectric cover is mounted within the chamber wall of the plasma reactor.

In former works about the pMRP its general performance was shown by CST simulations and measurements [28, 29]. However, an analytic model of the probe-plasma system to determine a simple relation between the measured resonance frequency and

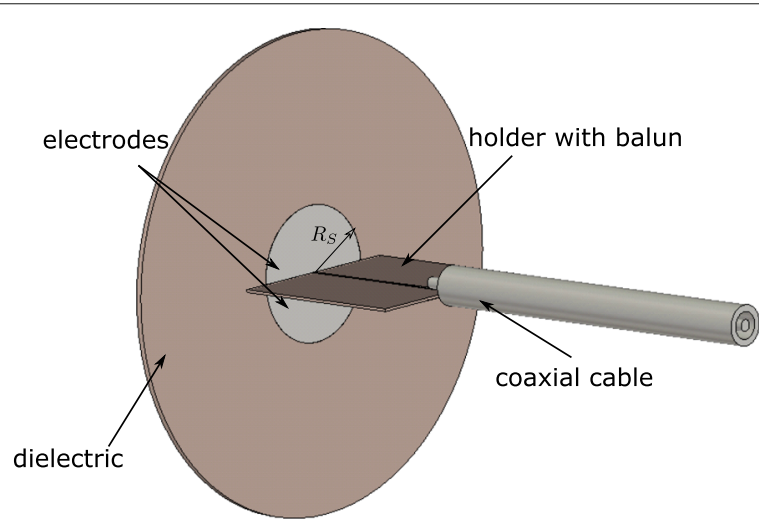

Fig. 2 Schematic of the pMRP (courtesy of D. Pohle, C. Schulz, and I. Rolfes), which consists of two half-disc electrodes with radius $R_{S}$ conducted to a tapered-balun and a coaxial cable. The electrodes are covered by a dielectric disc, which avoids a direct contact with the plasma 
the plasma frequency, which allows to measure the electron density, has not been derived, yet. Such a model will be presented in the next section.

\section{Model of the pMRP and its general admittance}

In a recent work a functional analytic description of APRS in an electrostatic approximation for probes in arbitrary geometry was derived and a general solution of the admittance was presented [27]. In this section we present the model of the pMRP and apply the general description to its specific geometry.

As depict in Fig. 3 the pMRP consists of two circular half-disc electrodes $\mathcal{E}_{1 / 2}$ with the radius $R_{S}$, which are perfectly integrated into the chamber wall. The electrodes are insulated to each other and to the grounded chamber wall. A rf signal is applied to each of the electrodes, but with a 180 degree phase shift to each other. To allow for analytic solutions, a dielectric $\mathcal{D}$ with thickness $d$ covers the probe and also the chamber wall (In reality only the electrodes are covered by the dielectric and the whole probe including the dielectric is integrated into the chamber wall as described in "Consequences of symmetry in MRP and pMRP design" section).

$\mathcal{V}=\mathcal{P} \cup \mathcal{S} \cup \mathcal{D}$ is the domain of the dynamical interaction between the probe and the plasma $\mathcal{P}$, where $\mathcal{S}$ is the sheath with thickness $\delta$. Caused by the circular half-disc electrodes a cylindrical coordinate system is chosen. The boundary $\partial \mathcal{V}$ of the dynamic interaction domain is then given by the electrode and wall surfaces at $z=0$ and at the surfaces $z \rightarrow \infty$ and $R=R_{\infty}$, where the dynamic interaction is assumed to vanish.

Within $\mathcal{S}$ and $\mathcal{P}$ the neutral gas is assumed as a stationary background. The same holds for the ions, because the frequency $\omega$ of the applied signal is much larger than the ion plasma frequency $\omega_{\mathrm{pi}}\left(\omega_{\mathrm{pi}} \ll \omega\right)$. Thus, the dynamical behavior of the plasma can be described by the cold plasma model in an electrostatic approximation for the electrons, given by the continuity equation and the generalized Ohm's law

$$
\begin{aligned}
& \frac{\partial \sigma_{\mathrm{e}}}{\partial t}=-\left.\vec{n} \cdot \vec{j}_{\mathrm{e}}\right|_{z=d+\delta}, \\
& \frac{\partial \rho_{\mathrm{e}}}{\partial t}=-\nabla \cdot \vec{j}_{\mathrm{e}}, \\
& \frac{\partial \overrightarrow{j_{\mathrm{e}}}}{\partial t}=-\varepsilon_{0} \omega_{\mathrm{pe}}^{2} \nabla \phi-v \vec{j}_{\mathrm{e}}-\varepsilon_{0} \omega_{\mathrm{pe}}^{2} \sum_{k=1}^{2} U_{k} \nabla \psi_{k} .
\end{aligned}
$$
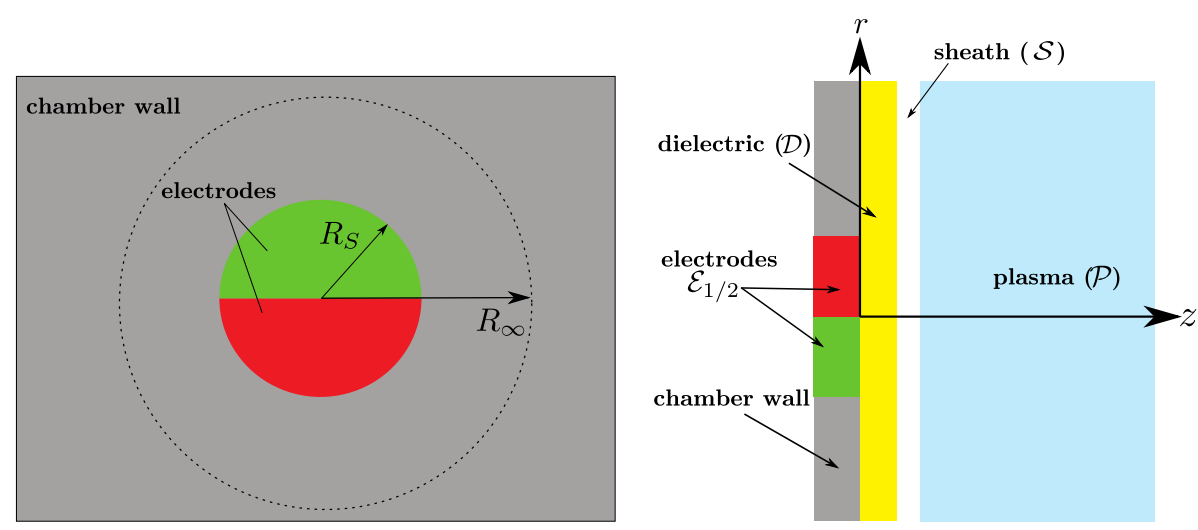

Fig. 3 The pMRP consists of two circular half-disc electrodes $\mathcal{E}_{1 / 2}$ with radius $R_{S}$ integrated into the chamber wall. A dielectric $\mathcal{D}$ covers the electrodes and the chamber wall. The sheath $\mathcal{S}$ in front of the dielectric separates it from the plasma $\mathcal{P}$ 
The physical variables are charge density $\rho_{\mathrm{e}}$ and current density $\overrightarrow{j_{\mathrm{e}}}$ of the electrons. $v$ represents the elastic collision frequency of the electrons with the neutral background gas and is assumed to be much smaller than the electron plasma frequency $\left(v \ll \omega_{\text {pe }}\right)$, which is typical for a low pressure plasma. Due to the complete electron depletion within the sheath, a surface charge density $\sigma_{\mathrm{e}}$ at the sheath edge $\mathcal{S K}$ has to be taken into account. $\phi$ is called inner potential and couples to the Poisson equation

$$
-\nabla \cdot\left(\varepsilon_{0} \varepsilon_{\mathrm{r}} \nabla \phi\right)= \begin{cases}0 & \vec{r} \in \mathcal{S} \cup \mathcal{D} \\ \sigma_{\mathrm{e}} & \vec{r} \in \mathcal{S K} \\ \rho_{\mathrm{e}} & \vec{r} \in \mathcal{P}\end{cases}
$$

with homogeneous boundary conditions. $\varepsilon_{\mathrm{r}}$, the respective permittivity, is given as 1 within $\mathcal{P}$ and $\mathcal{S}$ and as $\varepsilon_{\mathrm{D}}=$ const within $\mathcal{D}$. The excitation of the plasma due to the rf signal is represented by the applied voltages $U_{k}$ and the characteristic functions $\psi_{k}$, which fulfill Laplace's equation

$$
\nabla^{2} \psi_{k}=0
$$

with the boundary conditions

$$
\begin{aligned}
\left.\psi_{k}(\vec{r})\right|_{\vec{r} \in \mathcal{E}_{k^{\prime}}} & =\delta_{k k^{\prime}}, \\
\left.\psi_{k}(\vec{r})\right|_{z=0, r>R_{\mathrm{S}}} & =0, \\
\lim _{z \rightarrow \infty} \psi_{k}(\vec{r}) & =0 .
\end{aligned}
$$

$\delta_{k k^{\prime}}$ is the Kronecker Delta, which equals 1 if $k=k^{\prime}$ and 0 otherwise.

As presented in [27], the current at one electrode $\mathcal{E}_{1}$ is defined as the scalar product of an excitation vector $\left|e_{1}\right\rangle=\left(0,0,-\varepsilon_{0} \omega_{\mathrm{pe}}^{2} \nabla \psi_{1}\right)^{T}$ and the dynamical state vector $|z\rangle=$ $\left(\sigma_{\mathrm{e}}, \rho_{\mathrm{e}}, \overrightarrow{j_{\mathrm{e}}}\right)^{T}$

$$
i_{1}=\left\langle e_{1} \mid z\right\rangle=\sum_{k^{\prime}=1}^{2}\left\langle e_{1}\left|\left(i \omega-T_{\mathrm{C}}-T_{\mathrm{D}}\right)^{-1}\right| e_{k^{\prime}}\right\rangle U_{k^{\prime}}=\sum_{k^{\prime}=1}^{2} Y_{1 k^{\prime}} U_{k^{\prime}} .
$$

Based on the general solution of the dynamical state vector, $Y_{1 k^{\prime}}$ can be identified as the coupling admittance between the electrodes. It is given by

$$
Y_{1 k^{\prime}}=\left\langle e_{1}\left|\left(i \omega-T_{\mathrm{C}}-T_{\mathrm{D}}\right)^{-1}\right| e_{k^{\prime}}\right\rangle,
$$

where $T_{C}$ and $T_{D}$ are the conservative and dissipative operator, respectively. They and the corresponding scalar product are defined in Appendix A.

\section{Expanded admittance of the pMRP}

To compute specific spectra of the pMRP, its coupling admittance has to be expanded in an appropriate complete orthonormal basis. Since the eigenvalue of the operator $T_{D}$, which equals $v$, is assumed to be much smaller than the eigenvalues of the conservative operator $\mathrm{T}_{C}$, which are on the scale of $\omega_{\mathrm{pe}}$, a perturbation approach for operators can be applied [27]. Thus, the set of eigenstate vectors $\left\{\left|z_{n m}\right\rangle\right\}$ of $T_{C}$ is a suitable choice as a complete orthonormal basis (To make this section more readable, all derivations are shifted to the appendix.). It can be derived by solving the eigenvalue equation

$$
T_{\mathrm{C}}\left|z_{n m}\right\rangle=i \omega\left|z_{n m}\right\rangle \text {. }
$$


To solve this eigenvalue problem in cylindrical coordinates we expand all scalar functions into cylindrical harmonics

$$
\begin{gathered}
\sigma_{\mathrm{e}}(r, \varphi)=\sum_{n=1}^{\infty} \sum_{m=0}^{\infty} \sigma_{n m} J_{m}\left(k_{n m} r\right) e^{i m \varphi}, \\
\rho_{\mathrm{e}}(r, \varphi, z)=\sum_{n=1}^{\infty} \sum_{m=0}^{\infty} \rho_{n m}(z) J_{m}\left(k_{n m} r\right) e^{i m \varphi}, \\
\phi(r, \varphi, z)=\sum_{n=1}^{\infty} \sum_{m=0}^{\infty} \phi_{n m}(z) J_{m}\left(k_{n m} r\right) e^{i m \varphi},
\end{gathered}
$$

and the current density into vector cylindrical harmonics

$$
\vec{j}_{\mathrm{e}}(r, \varphi, z)=\sum_{n=1}^{\infty} \sum_{m=0}^{\infty}\left(j_{n m}^{(R)}(z) \vec{R}_{n m}+j_{n m}^{(\Phi)}(z) \vec{\Phi}_{n m}+j_{n m}^{(Z)}(z) \vec{Z}_{n m}\right) .
$$

$J_{m}\left(k_{n m} r\right)$ represent Bessel's functions of the $m$-th order. $k_{n m}=j_{m n} R_{\infty}^{-1}$ is its $n$-th eigenvalue connected to the $n$-th root $j_{m n}$ of the $m$-th Bessel function. The vector cylindrical harmonics are orthonormal on circular surfaces with the radius $R_{\infty}$. Their definitions and some properties are shown in Appendix B.

Similar to the calculations in [27], the authors assume a constant electron density in the bulk and thus $\omega_{\mathrm{pe}}=$ const to ensure square integrable eigenstate vectors. The normalized eigenstate vector to the eigenvalue

$$
\omega_{n m}= \pm \frac{1}{\sqrt{2}} \sqrt{\left(1-\left(1-\frac{2}{\varepsilon_{\mathrm{D}} \cosh \left(k_{n m} d\right)+1}\right) e^{-2 k_{n m} \delta}\right)} \omega_{\mathrm{pe}}= \pm \eta_{n m} \omega_{\mathrm{pe}}
$$

can then be derived

$$
\left|z_{n m}^{( \pm)}\right\rangle=\left(\begin{array}{c}
\phi_{n m}(z) J_{m}\left(k_{n m} r\right) e^{i m \varphi} \\
\pm \varepsilon_{0} \omega_{\mathrm{pe}}^{2} \frac{k_{n m}}{N_{\mathrm{Z}} \omega_{n m}}\left[\vec{\Phi}_{n m}-i \vec{Z}_{n m}\right] \phi_{n m}^{(\mathcal{P})}(z)
\end{array}\right) .
$$

It is important to note, that the inner potential is used in the eigenstate vector, but it couples unique to the surface charge density $\sigma_{\mathrm{e}}$ and the charge density $\rho_{\mathrm{e}}$ via Poisson's equation. $N_{Z}$ represents the normalization coefficient of the vector cylindrical harmonics (see Appendix B). To finalize the expansion of the coupling admittance, an explicit expression of the excitation vector is needed and is given as

$$
\left|e_{k}\right\rangle=\left(\begin{array}{c}
0 \\
-\frac{\varepsilon_{0} \omega_{p e}^{2}}{N_{Z}}\left[-\vec{\Phi}_{n m} i k_{n m} \psi_{n m}(z)+\vec{Z}_{n m} \frac{\mathrm{d} \psi_{n m}}{\mathrm{~d} z}\right]
\end{array}\right),
$$

including the $z$-dependent part of the characteristic functions $\psi_{n m}$.

Entering the completeness relation of the eigenstate vectors

$$
\sum_{n=1}^{\infty} \sum_{m=0}^{\infty}\left|z_{n m}^{(+)}\right\rangle\left\langle z_{n m}^{(+)}|+| z_{n m}^{(-)}\right\rangle\left\langle z_{n m}^{(-)}\right|=1
$$

twice and the excitation vector (18) into (10), the expanded coupling admittance of the pMRP reads as follows

$$
Y_{1 k^{\prime}}=\sum_{n=1}^{\infty} \sum_{m=0}^{\infty}\left[\frac{\varepsilon_{0} \omega_{\mathrm{pe}} k_{n m}}{N_{\mathrm{Z}}^{2} \eta_{n m}} B_{n m}^{(\mathcal{P})} e^{-2 k_{n m}(d+\delta)}\right]^{2} \frac{2 i \omega \beta_{n m}^{(1)} \beta_{n m}^{\left(k^{\prime}\right)}}{\omega_{\mathrm{pe}}^{2} \eta_{n m}^{2}+2 i \omega v_{n m}-\omega^{2}} .
$$


$B_{n m}^{(\mathcal{P})}, \beta_{n m}^{(1)}$, and $\beta_{n m}^{\left(k^{\prime}\right)}$ are integration constants, which are defined by the boundary and transition conditions of the eigenvalue problem (see Appendices $C$ and $E$ ). These constants include only geometric parameters of the probe. $v_{n m}=-\frac{1}{4} v$ are the matrix elements of the expanded dissipative operator $\mathrm{T}_{D}$ (see Appendix $\mathrm{F}$ ).

Finally, one can derive the admittance of the pMRP by entering the coupling admittance (20) into (9) and utilizing the 180 degree phase shift of the applied voltages $U_{1}=-U_{2}=U$ :

$$
Y=\sum_{n=1}^{\infty} \sum_{\tilde{m}=0}^{\infty} j_{2 \tilde{m}+1, n} J_{2(\tilde{m}+1)}^{2}\left(j_{2 \tilde{m}+1, n}\right) e^{-2 k_{n, 2 \tilde{m}+1}(d+\delta)} \frac{4 \pi \varepsilon_{0} \omega_{\mathrm{pe}}^{2} R_{\infty} i \omega \beta_{n, 2 \tilde{m}+1}^{(1)^{2}}}{2 \omega_{p e}^{2} \eta_{n, 2 \tilde{m}+1}^{2}-i \omega \nu-2 \omega^{2}}
$$

The admittance vanishes for even $m$, which yields a final sum over odd $m=2 \tilde{m}+1$.

\section{Converged spectra of the pMRP}

The admittance of the pMRP, derived within the last section, can be used to plot and analyze its spectrum. However, the spectrum is not given by an infinite number of discrete resonance modes, like the spectrum of probes with a spherical probe tip [27], because the electrode geometry of the pMRP is not represented by Delta functions in the corresponding Fourier space. This means, that the admittance of the pMRP will have a spectrum with a broad resonance as a superposition of all addends in the double series (21). Thus, to determine explicit spectra, an approximated admittance with truncated sums is needed.

Within this section we analyze the convergence behavior of the pMRP spectrum dependent on three parameters: the radius of the boundary surface $R_{\infty}$, the upper boundary of the inner sum $\tilde{M}_{\max }$ and the upper boundary of the outer sum $N_{\max }$. All other parameters are given by the geometry of the probe or the plasma itself and influence just the position of the resonance. In order to compare the determined spectra, we choose parameters of the pMRP taken from a recent published paper [29]: $R_{\mathrm{S}}=3 \mathrm{~mm}, d=0.4 \mathrm{~mm}, \varepsilon_{\mathrm{D}}=3.55$, $\delta=0.3 \mathrm{~mm}, \omega_{\mathrm{pe}}=2 \pi \cdot 2.8 \cdot 10^{9} \mathrm{~s}^{-1}$ and $\nu=0.015 \omega_{\mathrm{pe}}$.

Figure 4 shows four different spectra to demonstrate the influence of $R_{\infty}$ for 5 (bold orange line), 10 (dashed red line), 20 (dot-dashed blue line), and 40 (dotted black line) times the probe radius $R_{\mathrm{S}}$. $\tilde{M}_{\max }=10$ and $N_{\max }=200$ are set to large numbers, to focus only on the influence of $R_{\infty}$. If $R_{\infty}$ is too small, single peaks can be observed in the spectra. They correspond to certain eigenvalues, but have no physical meaning. The larger $R_{\infty}$ the smoother the spectrum gets and one broad resonance peak is formed. Above $R_{\infty}=40 R_{\mathrm{S}}$ the spectrum is practically converged. Thus, $R_{\infty}=40 R_{\mathrm{S}}$ represents the minimal radius for the boundary surface and will be used in further calculations within this manuscript.

The influence of $\tilde{M}_{\max }$ is not tremendous. It is shown for $\tilde{M}_{\max }$ equal to 0,1 , and 2 with $N_{\max }=200$ in Fig. 5. A small difference in the height of the peaks and in the behavior above $\omega=0.6 \omega_{\text {pe }}$ can be observed in the spectra on the left hand side for $\tilde{M}_{\max }=$ 0 (dashed blue line) and $\tilde{M}_{\max }=1$ (bold red line). Increasing $\tilde{M}_{\max }$ to 2 (see Fig. 5, right, dotted) changes just the behavior above $\omega=0.6 \omega_{\text {pe }}$, which can be interpreted as contribution of higher modes, but the position and the height of the main peak remain the same. A further increase of $\tilde{M}_{\max }$ shows no difference within the spectra and can be neglected. Since the main resonance is not influenced by larger values of $\tilde{M}_{\max }$, we define it to 1 for further calculations. 


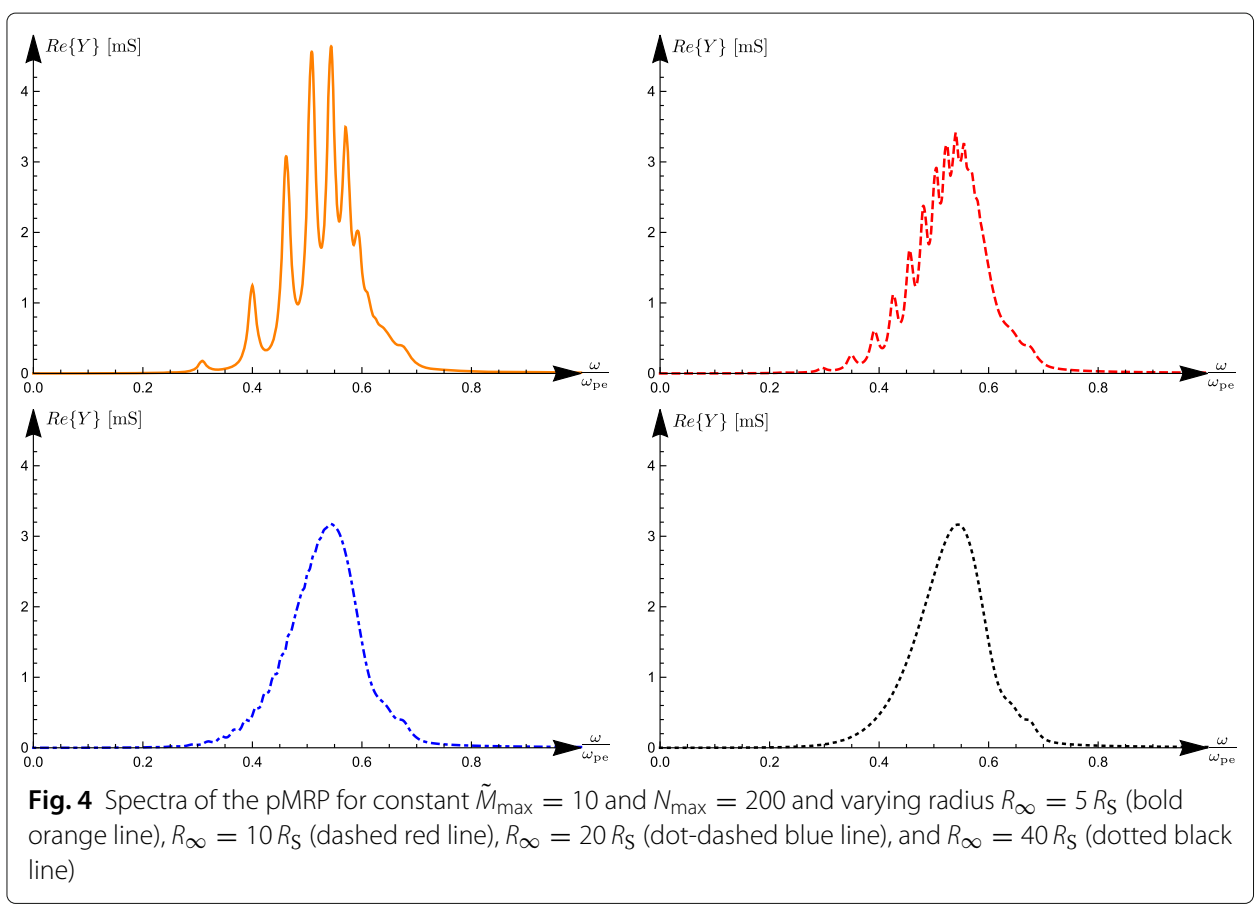

A strong influence is given by the outer sum truncated with $N_{\max }$, which is shown in Fig. 6. On the left hand side the height and also the position of the peaks differ for $N_{\max }$ equal to 50 (dashed orange line) and 75 (dot-dashed blue line). The spectra on the right hand side for $N_{\max }$ equal to 100 (bold red line) and 125 (dotted black line) differ just slightly for values above $\omega=0.6 \omega_{\text {pe }}$, but the height and the position of the peak remain the same. A further increase of $N_{\max }$ leads to identical spectra, which defines $N_{\max }=125$ as smallest upper boundary of the outer sum for practically converged spectra.

Thus, practically converged spectra for the pMRP can be determined for $\tilde{M}_{\max }=1$, $N_{\max }=125$, and $R_{\infty}=40 R_{\mathrm{S}}$. In Fig. 7 (left) converged spectra for three different probe radii $R_{\mathrm{S}}=2 \mathrm{~mm}$ (dotted orange line), $R_{\mathrm{S}}=3 \mathrm{~mm}$ (bold red line), and $R_{\mathrm{S}}=4 \mathrm{~mm}$ (dashed black line) are depicted. The corresponding resonance frequencies are $\omega_{\mathrm{r}}=0.598 \omega_{\mathrm{pe}}$, $\omega_{\mathrm{r}}=0.542 \omega_{\mathrm{pe}}$, and $\omega_{\mathrm{r}}=0.497 \omega_{\mathrm{pe}}$, respectively. They represent the proportional relations between the resonance and the plasma frequency, which are required to determine the electron density from a measured resonance. They are in good agreement with the CST simulations presented in [29] for the chosen probe and plasma parameters.
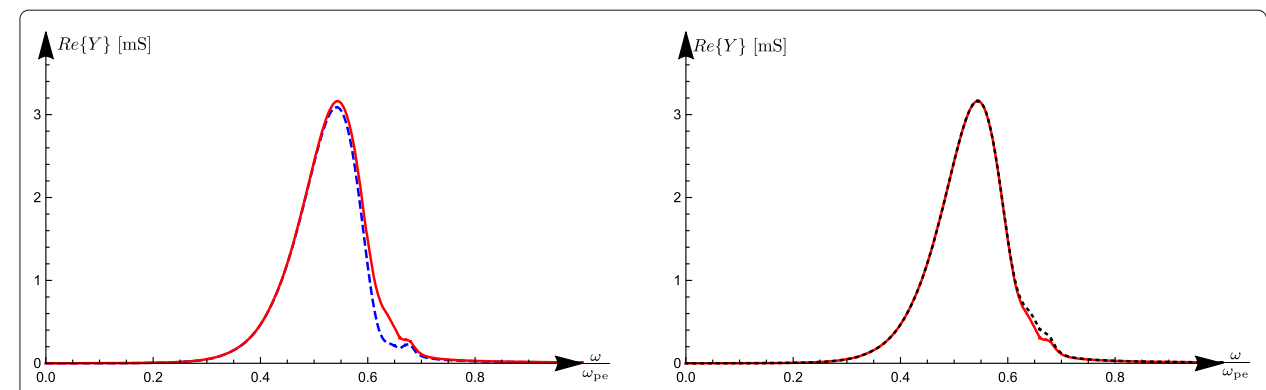

Fig. 5 Spectra of the pMRP for constant $N_{\max }=200$ and $R_{\infty}=40 R_{S}$ and varying $\tilde{M}_{\max }=0$ (dashed blue line), $\tilde{M}_{\max }=1$ (bold red line), and $\tilde{M}_{\max }=2$ (dotted) 


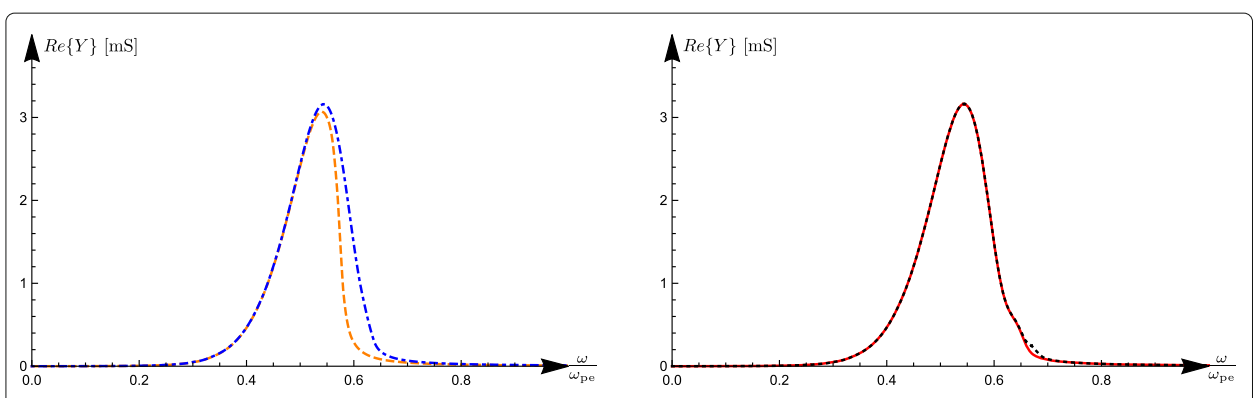

Fig. 6 Spectra of the pMRP for constant $\tilde{M}_{\max }=1$ and $R_{\infty}=40 R_{\mathrm{s}}$ and varying $N_{\max }=50$ (dashed orange line), $N_{\max }=75$ (dot-dashed blue line), $N_{\max }=100$ (bold red line) and $N_{\max }=125$ (dotted black line)

As presented in ref. [29], the different probe radii are required to cover different frequency ranges. Within these ranges the relation between the plasma frequency $f_{\mathrm{p}}$ and the measured resonance frequency $f_{\mathrm{r}}$ are plotted in Fig. 7 (right). These frequency ranges cover the following ranges of electron densities given in $10^{16} \mathrm{~m}^{-3}: 1.8-8.4,6-16$, and $13-26$. The results show the same trend, but differ slightly in the proportionality. The reason for that can be explained by the differences between the analytic model in the electrostatic approximation and the full three dimensional electromagnetic numerical simulations.

\section{Conclusion}

Within this work the authors presented the first analytic model of the pMRP and derived the general admittance of the probe-plasma system by means of functional analytic methods. To determine an explicit expression of the admittance a complete orthonormal set of basis functions was derived by the eigenvalue problem of the conservative operator $T_{C}$. They are based on cylindrical harmonic functions, due to the cylindrical geometry of the calculation domain.

The explicit admittance is represented by an analytic expression, but it is given by an infinite expansion and has to be truncated to determine specific spectra of the pMRP. Due to that, a convergence analysis is presented to define the minimum values of the parameters $R_{\infty}=40 R_{S}, N_{\max }=125$, and $\tilde{M}_{\max }=1$. They influence the convergence behavior.

In the converged spectra a unique resonance peak is observable, similar to the spectra of the spherical impedance probe (sIP) or the MRP. However, its half width is much larger.
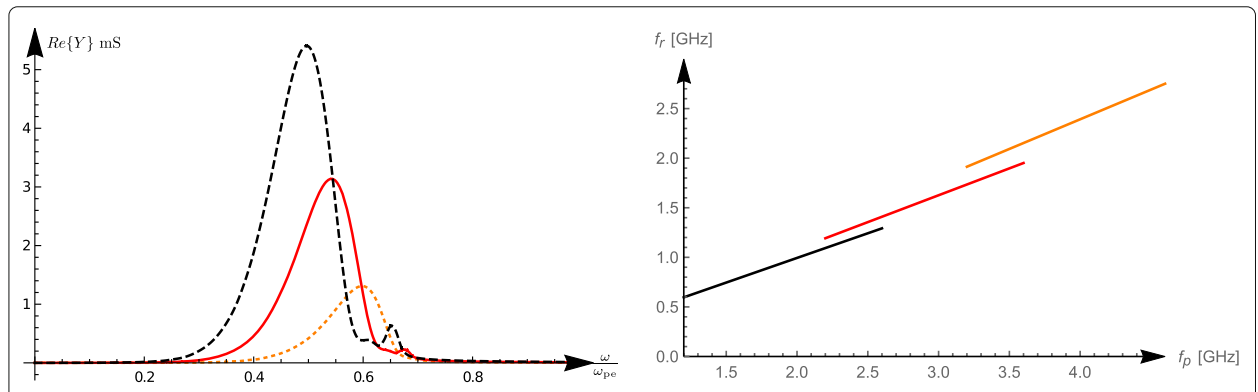

Fig. 7 Converged spectra of the pMRP (left) and relations between the resonance and the plasma frequencies (right) for different probe radii: $R_{S}=2 \mathrm{~mm}$ (dotted orange line), $R_{S}=3 \mathrm{~mm}$ (bold red line), and $R_{S}=4 \mathrm{~mm}$ (dashed black line) 
This broader resonance peak is not caused by stronger damping or an additional damping mechanism like kinetic damping. It is due to the fact, that the eigenfunctions of the electrodes geometry are not represented by Delta functions in the corresponding Fourier space as in spherical geometry.

Based on the converged spectra resonance frequencies for probes with different probe radii can be determined, which are in good agreement with former CST simulations [29]. As proposed in this reference probes with different probe radii should be used to cover different frequency ranges. Within these frequency ranges the analytically determined resonance frequencies in this mansuscript are larger than that from the simulations. This is caused by a metallic adapter ring, which serves as planar impedance matching in the pMRP design. First simulations show, that a decrease of the inner radius of this ring shifts the resonances to larger frequencies, which leads to a better agreement with the analytic model.

Based on the presented model the pMRP can be used for measurements of the electron density by means of the proportional relation $\omega_{\mathrm{r}} \propto \omega_{\mathrm{pe}}$. It is an excellent candidate to monitor and/or control plasma processes. As a next step further detailed comparisons of the presented model in an electrostatic approximation to full three dimensional electromagnetic simulations are planed to analyze the differences within the model and the simulation results. Furthermore, measurements with the pMRP compared to other diagnostic tools will follow.

\section{Appendix A: operators and scalar product}

The general definitions of the conservative and dissipative operator and the scalar product are given in [26]. Here, we define these operators and the scalar product for the geometry of the pMRP. The operators are given by

$$
\begin{aligned}
& T_{\mathrm{C}}|z\rangle=\left(-\left.\vec{n} \cdot \overrightarrow{j_{\mathrm{e}}}\right|_{z=d+\delta},-\nabla \cdot \vec{j}_{\mathrm{e}},-\varepsilon_{0} \omega_{\mathrm{pe}}^{2} \nabla \phi\right)^{T}, \\
& T_{\mathrm{D}}|z\rangle=\left(0,0,-v \overrightarrow{\dot{j}_{\mathrm{e}}}\right)^{T},
\end{aligned}
$$

and the scalar product between two different state vectors is defined as

$$
\begin{aligned}
\left\langle z^{\prime} \mid z\right\rangle= & \int_{0}^{\infty} \int_{0}^{2 \pi} \int_{0}^{R_{\infty}} \varepsilon_{0} \varepsilon_{\mathrm{r}} \nabla \phi^{\prime *} \cdot \nabla \phi r \mathrm{~d} r \mathrm{~d} \varphi \mathrm{d} z \\
& +\int_{d+\delta}^{\infty} \int_{0}^{2 \pi} \int_{0}^{R_{\infty}} \frac{1}{\varepsilon_{0} \omega_{\mathrm{pe}}^{2}} \vec{j}_{\mathrm{e}}^{\prime *} \cdot \vec{j}_{\mathrm{e}} r \mathrm{~d} r \mathrm{~d} \varphi \mathrm{d} z .
\end{aligned}
$$

\section{Appendix B: vector cylindrical harmonics}

The vector cylindrical harmonics used in this manuscript are defined as

$$
\begin{aligned}
& \vec{Z}_{n m}=Z_{n m} \vec{e}_{z}=\frac{J_{m}\left(k_{n m} r\right) e^{i m \varphi}}{\sqrt{\pi} R_{\infty} J_{m+1}\left(j_{m n}\right)} \vec{e}_{z}=N_{\mathrm{Z}} J_{m}\left(k_{n m} r\right) e^{i m \varphi} \vec{e}_{z}, \\
& \vec{R}_{n m}=\frac{1}{k_{n m}} \vec{L} Z_{n m}, \\
& \vec{\Phi}_{n m}=\vec{e}_{z} \times \vec{R}_{n m} .
\end{aligned}
$$

$\vec{L}=-i \vec{e}_{z} \times \vec{\nabla}$ is a rotation operator motivated by the angular momentum. These functions build an orthonormal basis on the circular surfaces with radius $R_{\infty}$ and fulfill the following orthogonal relations: 


$$
\begin{aligned}
& \int_{0}^{2 \pi} \int_{0}^{R_{\infty}} \vec{Z}_{n m}^{*} \cdot \vec{Z}_{n^{\prime} m^{\prime}} r \mathrm{~d} r \mathrm{~d} \varphi=\delta_{n n^{\prime}} \delta_{m m^{\prime}}, \\
& \int_{0}^{2 \pi} \int_{0}^{R_{\infty}} \vec{R}_{n m}^{*} \cdot \vec{R}_{n^{\prime} m^{\prime}} r \mathrm{~d} r \mathrm{~d} \varphi=\delta_{n n^{\prime}} \delta_{m m^{\prime}}, \\
& \int_{0}^{2 \pi} \int_{0}^{R_{\infty}} \vec{\Phi}_{n m}^{*} \cdot \vec{\Phi}_{n^{\prime} m^{\prime}} r \mathrm{~d} r \mathrm{~d} \varphi=\delta_{n n^{\prime}} \delta_{m m^{\prime}}, \\
& \int_{0}^{2 \pi} \int_{0}^{R_{\infty}} \vec{Z}_{n m}^{*} \cdot \vec{R}_{n^{\prime} m^{\prime}} r \mathrm{~d} r \mathrm{~d} \varphi=0, \\
& \int_{0}^{2 \pi} \int_{0}^{R_{\infty}} \vec{Z}_{n m}^{*} \cdot \vec{\Phi}_{n^{\prime} m^{\prime}} r \mathrm{~d} r \mathrm{~d} \varphi=0, \\
& \int_{0}^{2 \pi} \int_{0}^{R_{\infty}} \vec{\Phi}_{n m}^{*} \cdot \vec{R}_{n^{\prime} m^{\prime}} r \mathrm{~d} r \mathrm{~d} \varphi=0 .
\end{aligned}
$$

\section{Appendix C: derivation of the eigenstate vector}

The eigenvalue problem (11) expanded into the scalar and vector cylindrical harmonics reads as follows

$$
\begin{aligned}
i \omega \sigma_{n m} & =-\left.j_{n m}^{(z)}\right|_{z=d+\delta}, \\
i \omega \rho_{n m} & =\frac{\partial^{2} \phi_{n m}}{\partial z^{2}}-i k_{n m}^{2} \phi_{n m}(z), \\
i \omega j_{n m}^{(R)} & =0, \\
i \omega j_{n m}^{(\Phi)} & =\frac{i \varepsilon_{0} \omega_{\mathrm{pe}}^{2} k_{n m}}{N_{\mathrm{Z}}} \phi_{n m}, \\
i \omega j_{n m}^{(Z)} & =-\frac{\varepsilon_{0} \omega_{\mathrm{pe}}^{2}}{N_{\mathrm{Z}}} \frac{\partial \phi_{n m}}{\partial z} .
\end{aligned}
$$

Applying the expansion to Poisson's equation simplifies it to the $z$-component of the inner potential

$$
-\varepsilon_{0}\left[\frac{\partial^{2} \phi_{n m}}{\partial z^{2}}-k_{n m}^{2} \phi_{n m}\right]= \begin{cases}0 & z \in \mathcal{S} \cup \mathcal{D} \\ \sigma_{e} & z \in \mathcal{S} \mathcal{K} \\ \rho_{e} & z \in \mathcal{P}\end{cases}
$$

with the boundary conditions

$$
\phi_{n m}^{(\mathcal{D})}(0)=0 \quad \text { and } \quad \lim _{z \rightarrow \infty} \phi_{n m}^{(\mathcal{P})}(z)=0,
$$

and the transition conditions

$$
\begin{aligned}
& \phi_{n m}^{(\mathcal{D})}(d)-\phi_{n m}^{(\mathcal{S})}(d)=0, \\
& \phi_{n m}^{(\mathcal{S})}(d+\delta)-\phi_{n m}^{(\mathcal{P})}(d+\delta)=0, \\
& {\left[\frac{\mathrm{d} \phi_{n m}^{(\mathcal{S})}}{\mathrm{d} z}-\varepsilon_{\mathrm{D}} \frac{\mathrm{d} \phi_{n m}^{(\mathcal{D})}}{\mathrm{d} z}\right]_{z=d} }=0, \\
& {\left[\left(1-\frac{\omega_{\mathrm{pe}}^{2}}{\omega^{2}}\right) \frac{\mathrm{d} \phi_{n m}^{(\mathcal{P})}}{\mathrm{d} z}-\frac{\mathrm{d} \phi_{n m}^{(\mathcal{S})}}{\mathrm{d} z}\right]_{z=d+\delta}=0 . }
\end{aligned}
$$


Within the plasma domain $\mathcal{P}$ the dynamic Eqs. (34) to (38) can be simplified to one equation for the charge density $\rho_{n m}$, which can be entered into (39) to find

$$
\left[1-\frac{\omega_{\mathrm{pe}}^{2}}{\omega^{2}}\right]\left[\frac{\mathrm{d}^{2} \phi_{n m}^{(\mathcal{P})}}{\mathrm{d} z^{2}}-k_{n m}^{2} \phi_{n m}^{(\mathcal{P})}\right]=0 .
$$

Relevant frequencies have to fulfill $\omega \neq \omega_{\text {pe }}$, and thus the right bracket of Eq. (45) has to be equal to zero, which represents the expanded Laplace equation. Also within the sheath $\mathcal{S}$ and the dielectric $\mathcal{D}$ Laplace's equation holds. Its general solution for the inner potential of all domains $\phi_{n m}^{(\mathcal{D}, \mathcal{S}, \mathcal{P})}$ is given by

$$
\phi_{n m}^{(\mathcal{D}, \mathcal{S}, \mathcal{P})}(z)=A_{n m}^{(\mathcal{D}, \mathcal{S}, \mathcal{P})} e^{k_{n m} z}+B_{n m}^{(\mathcal{D}, \mathcal{S}, \mathcal{P})} e^{-k_{n m} z} .
$$

Applying the transition and boundary conditions five of the six constants and the eigenvalues $\omega_{n m}$ of $T_{\mathrm{C}}$ in cylindrical coordinates can be determined

$$
\begin{aligned}
& B_{n m}^{(\mathcal{D})}=-A_{n m}, \\
& A_{n m}^{(\mathcal{S})}=\frac{1}{2}\left[1+e^{-2 d k_{n m}}\left(\varepsilon_{\mathrm{D}}-1\right)+\varepsilon_{\mathrm{D}}\right] A_{n m}, \\
& B_{n m}^{(\mathcal{S})}=\frac{1}{2}\left[-1-e^{2 d k_{n m}}\left(\varepsilon_{\mathrm{D}}-1\right)-\varepsilon_{\mathrm{D}}\right] A_{n m}, \\
& A_{n m}^{(\mathcal{P})}=0, \\
& B_{n m}^{(\mathcal{P})}=-\frac{1}{2}\left[\left(1+\varepsilon_{\mathrm{D}}\right)\left(1-e^{2 k_{n m}(d+\delta)}\right)+\left(\varepsilon_{\mathrm{D}}-1\right)\left(e^{2 d k_{n m}}-e^{2 \delta k_{n m}}\right)\right] A_{n m} .
\end{aligned}
$$

$A_{n m}$, the last constant, can be derived by normalization of the eigenstate vector.

\section{Appendix D: normalization}

The corresponding norm of an eigenstate vector is defined by the square root of the scalar product of two identical eigenstate vectors $\left|z_{n m}\right\rangle$. In cylindrical geometry the scalar product of these eigenstate vectors reads as follows

$$
\begin{aligned}
\left\langle z_{n m} \mid z_{n m}\right\rangle= & \frac{1}{N_{\mathrm{Z}}^{2}} \int_{0}^{\infty} \varepsilon_{0} \varepsilon_{\mathrm{r}}\left[\left(\frac{\mathrm{d} \phi_{n m}(z)}{\mathrm{d} z}\right)^{2}+k_{n m}^{2} \phi_{n m}^{2}(z)\right] \mathrm{d} z \\
& +\int_{d+\delta}^{\infty} \frac{1}{\varepsilon_{0} \omega_{\mathrm{pe}}^{2}}\left[\left|j_{n m}^{(R)}(z)\right|^{2}+\left|j_{n m}^{(\Phi)}(z)\right|^{2}+\left|j_{n m}^{(Z)}(z)\right|^{2}\right] \mathrm{d} z .
\end{aligned}
$$

Fulfilling the condition $\left\|z_{n m}\right\|=\sqrt{\left\langle z_{n m} \mid z_{n m}\right\rangle}=1$, the last constant $A_{n m}$ can be determined

$$
A_{n m}=\sqrt{\frac{N_{\mathrm{Z}}^{2} e^{-2 k_{n m} d}\left[\varepsilon_{\mathrm{D}}-1+e^{2 k_{n m} d}\left(\varepsilon_{\mathrm{D}}+1\right)\right]^{-1}}{k_{n m} \varepsilon_{0}\left[\left(1+\varepsilon_{\mathrm{D}}\right)\left(1-e^{2 k_{n m}(d+\delta)}\right)+\left(\varepsilon_{\mathrm{D}}-1\right)\left(e^{2 d k_{n m}}-e^{2 \delta k_{n m}}\right)\right]}} .
$$

\section{Appendix E: derivation of the excitation vector}

The excitation vector $\left|e_{k}\right\rangle$ contains the characteristic functions $\psi_{k}$, which follow Laplace's equation

$$
\nabla \cdot\left(\varepsilon_{0} \varepsilon_{D} \nabla \psi_{k}=0\right) \text { with } \lim _{z \rightarrow \infty} \psi_{k}=0 \text { and }\left.\psi_{k}\right|_{\mathcal{E}_{k^{\prime}}}=\delta_{k k^{\prime}} .
$$


Similar to the derivation of the eigenstate vectors we expand the characteristic functions in cylindrical harmonics and determine the solution in $z$-direction:

$$
\psi_{n m}= \begin{cases}\alpha^{(\mathcal{D})} e^{k_{n m} z}+\beta^{(\mathcal{D})} e^{-k_{n m} z} & z \in \mathcal{D} \\ \beta^{(\mathrm{vac})} e^{-k_{n m} z} & z \in \mathcal{S} \cup \mathcal{P} .\end{cases}
$$

The characteristic functions have to fulfill the following transition conditions:

$$
\psi_{n m}^{(\mathcal{D})}(d)=\psi_{n m}^{(\mathrm{vac})}(d) \quad,\left.\quad \varepsilon_{\mathrm{D}} \frac{\partial}{\partial z} \psi_{n m}^{(\mathcal{D})}\right|_{d}=\left.\frac{\partial}{\partial z} \psi_{n m}^{(\mathrm{vac})}\right|_{d} .
$$

Based on these equations it is possible to determine the constants $\alpha^{(\mathcal{D})}$ and $\beta^{(\mathcal{D})}$ dependent on $\beta^{(\mathrm{vac})}=\beta_{n m}^{(k)}$

$$
\begin{aligned}
\alpha^{(\mathcal{D})} & =\frac{e^{-2 k_{n m} d}\left(\varepsilon_{\mathrm{D}}-1\right)}{2 \varepsilon_{\mathrm{D}}} \beta_{n m}^{(k)}, \\
\beta^{(\mathcal{D})} & =\frac{\varepsilon_{\mathrm{D}}+1}{2 \varepsilon_{\mathrm{D}}} \beta_{n m}^{(k)} .
\end{aligned}
$$

The general excitation vector can then be determined to

$$
\left|e_{k}\right\rangle=\sum_{n, m}\left(0,0, \varepsilon_{0} \omega_{\mathrm{pe}}^{2} \beta_{n m}^{(k)} k_{n m} e^{-k_{n m} z}\left[\vec{\Phi}_{n m} i-\vec{Z}_{n m}\right]\right) .
$$

The remaining coefficient $\beta_{n m}^{(k)}$ can be calculated by the boundary condition $\psi_{n m}^{(k)}(0)=\delta_{k k^{\prime}}$ at the electrodes $\mathcal{E}_{k}$. Applying the orthogonality relation of the Bessel functions we find

$$
\begin{aligned}
& \beta_{n m}^{(1)}=\frac{i \varepsilon_{\mathrm{D}}\left(R_{\mathrm{S}} N_{\mathrm{Z}}\right)^{2}\left(\frac{k_{n m} R_{\mathrm{S}}}{-2}\right)^{m}\left((-1)^{m}-1\right) \Gamma\left(\frac{m}{2}\right){ }_{1} F_{2}\left(\frac{m}{2}+1 ; \frac{m}{2}+2, m+1 ; \frac{-R_{\mathrm{S}}^{2} k_{n m}^{2}}{4}\right)}{2\left[\left(\varepsilon_{\mathrm{D}}-1\right) e^{-2 k_{n m} d}+\varepsilon_{\mathrm{D}}+1\right]}, \\
& \beta_{n m}^{(2)}=(-1)^{m} \beta_{n m}^{(1)},
\end{aligned}
$$

for the pMRP. Here ${ }_{1} F_{2}\left(a ;\left\{b_{1}, b_{2}\right\} ; z\right)$ is the generalized hypergeometric function and $\Gamma(z)$ the gamma function.

\section{Appendix F: matrix elements of the operators $T_{C}$ and $T_{D}$}

Due to the fact, that $\left|z_{n m}\right\rangle$ are the eigenstate vectors of the conservative operator $T_{C}$, its matrix elements can be calculated as

$$
\left\langle z_{n^{\prime} m^{\prime}}\left|T_{C}\right| z_{n m}\right\rangle=i \omega_{n m} \delta_{n n^{\prime}} \delta_{m m^{\prime}} .
$$

The dissipative operator applied to an eigenstate vector is given by

$$
T_{\mathrm{D}}\left|z_{n m}\right\rangle=\left(0,0,-\frac{\nu \varepsilon_{0} \omega_{\mathrm{pe}}^{2}}{N_{\mathrm{Z}} \omega_{n m}}\left[\vec{\Psi}_{n m} k_{n m} \phi_{n m}^{(\mathcal{P})}+\vec{Z}_{n m} i \frac{\mathrm{d} \phi_{n m}^{(\mathcal{P})}}{\mathrm{d} z}\right]\right)
$$

and leads to

$$
\begin{aligned}
\left\langle z_{n m}\left|T_{D}\right| z_{n^{\prime} m^{\prime}}\right\rangle & =-\frac{v\left(\varepsilon_{0} \omega_{\mathrm{pe}}^{2}\right)^{2}}{N_{\mathrm{Z}^{2}}^{2} \omega_{n^{\prime} m^{\prime}} \omega_{n m}} \int_{d+\delta}^{\infty} \frac{1}{\varepsilon_{0} \omega_{\mathrm{pe}}^{2}}\left[k_{n^{\prime} m^{\prime}} k_{n m} \phi_{n^{\prime} m^{\prime}}^{(\mathcal{P})} \phi_{n m}^{(\mathcal{P})}+\frac{\mathrm{d} \phi_{n^{\prime} m^{\prime}}^{(\mathcal{P})}}{\mathrm{d} z} \frac{\mathrm{d} \phi_{n m}^{(\mathcal{P})}}{\mathrm{d} z}\right] \mathrm{d} z \delta_{n n^{\prime}} \delta_{m m^{\prime}} \\
& =-\frac{v \varepsilon_{0} \omega_{\mathrm{pe}}^{2} B_{n m}^{(\mathcal{P})^{2}} k_{n m}}{2 N_{\mathrm{Z}}^{2} \omega_{n m}^{2}} e^{-2 k_{n m}(d+\delta)}=-\frac{v}{4}=v_{n m} .
\end{aligned}
$$




\section{Acknowledgments}

The authors acknowledge support by the internal funding of the Leuphana University Lüneburg and the German Research Foundation via the project OB 469/1-1. Gratitude is expressed to J. Gong, D.-B. Grys, M. Lapke, M. Oberberg, D. Pohle, C. Schulz, J. Runkel, R. Storch, T. Styrnoll, S. Wilczek, T. Mussenbrock, P. Awakowicz, T. Musch, and I. Rolfes, who are or were part of the MRP-Team at Ruhr University Bochum. Explicit gratitude is expressed to R.P. Brinkmann for fruitful discussions.

\section{Funding}

German Research Foundation via the project OB 469/1-1

Authors' contributions

Both authors read and approved the final manuscript.

Ethics approval and consent to participate

Not applicable.

\section{Consent for publication}

Not applicable.

\section{Competing interests}

The authors declare that they have no competing interests.

\section{Publisher's Note}

Springer Nature remains neutral with regard to jurisdictional claims in published maps and institutional affiliations.

\section{Received: 25 January 2018 Accepted: 29 June 2018}

Published online: 09 August 2018

\section{References}

1. Lapke M, et al. (2011) The multipole resonance probe: characterization of a prototype. Plasma Sources Sci Technol 20:042001

2. Takayama K, Ikegami H, Miyazaki S (1960) Plasma resonance in a radio-frequency probe. Phys Rev Let 5:238

3. Messiaen AM, Vandenplas PE (1966) High-frequency dielectric resonance probe for the measurement of plasma densities. J Appl Phys 37:1718

4. Waletzko JA, Bekefi G (1967) RF admittance measurements of a slotted-sphere antenna immersed in a plasma. Radio Sci 2:489

5. Vernet N, Manning R, Steinberg JL (1975) The impedance of a dipole antenna in the ionosphere: 1. Experimental study. Radio Sci 10:517

6. Blackwell DD, Walker DN, Amatucci WE (2005) Measurement of absolute electron density with a plasma impedance probe. Rev Sci Instrum 76:023503

7. Kokura H, Nakamura K, Ghanashev IP, Sugai H (1999) Plasma Absorption Probe for Measuring Electron Density in an Environment Soiled with Processing Plasmas. Japan J Appl Phys 38:5262

8. Scharwitz C, Böke M, Winter J, Lapke M, Mussenbrock T, Brinkmann RP (2009) Practical implementation of a two-hemisphere plasma absorption probe. Appl Phys Lett 94:011502

9. Lapke M, Mussenbrock T, Brinkmann RP (2008) The multipole resonance probe: A concept for simultaneous determination of plasma density, electron temperature, and collision rate in low-pressure plasmas. Appl Phys Lett 93:051502

10. Fejer JA (1964) Interaction of an antenna with a hot plasma and the theory of resonance probes. Radio Sci 68D:1171

11. Harp RS (1964) The behavior of the resonance probe in a plasma. Appl Phys Lett 4:186

12. Harp RS, Crawford FW (1964) Characteristics of the plasma resonance probe. J Appl Phys 35:3436

13. Dote T, Ichimiya T (1965) Characteristics of resonance probes. J Appl Phys 36:1866

14. Kostelnicek RJ (1968) An investigation of boundary theories for the resonance probe. Radio Sci 3:319

15. Cohen AJ, Bekefi G (1971) Linear and nonlinear response of a plasma sheath to radio frequency potentials. Phys Fluids 14:1512

16. Tarstrup J, Heikkila WJ (1972) The impedance characteristic of a spherical probe in an isotropic plasma. Radio Sci 4:493

17. Aso T (1973) Impedance of an ion-sheathed spherical probe in a warm, isotropic plasma. Radio Sci 8:139

18. Bantin CC, Balmain KG (1974) Radio-frequency probes in a nonlinear isotropic plasma. Can J Phys 52:291

19. Dine S, Booth JP, Curley GA, Corr CS, Jolly J, Guillon J (2005) A novel technique for plasma density measurement using surface-wave transmission spectra. Plasma Scources Sci Technol 14:777

20. Walker DN, Fernsler RF, Blackwell DD, Amatucci WE, Messer SJ (2006) On collisionless energy absorption in plasmas: Theory and experiment in spherical geometry. Phys Plasmas 13:032108

21. Lapke M, Mussenbrock T, Brinkmann RP, Scharwitz C, Böke M, Winter J (2007) Modeling and simulation of the plasma absorption probe. Appl Phys Lett 90:121502

22. Xu J, Nakamura K, Zhang Q, Sugai H (2009) Simulation of resistive microwave resonator probe for high-pressure plasma diagnostics. Plasma Sources Sci Technol 18:045009

23. Xu J, Shi J, Zhang J, Zhang Q, Nakamura K, Sugai H (2010) Advanced high-pressure plasma diagnostics with hairpin resonator probe surrounded by film and sheath. Chinese Phys B 19:075206

24. Li B, Li H, Chen Z, Xie J, Feng G, Liu W (2010) Experimental and simulational studies on the theoretical model of the plasma absorption probe. Plasma Sci Technol 12:513

25. Linag I, Nakamura K, Sugai H (2011) Modeling microwave resonance of curling probe for density measurements in reactive plasmas. Appl Phys Express 4:066101 
26. Lapke M, Oberrath J, Mussenbrock T, Brinkmann RP (2013) Active plasma resonance spectroscopy: a functional analytic description. Plasma Scources Sci Technol 22:025005

27. Oberrath J, Brinkmann RP (2014) Active plasma resonance spectroscopy: eigenfunction solutions in spherical geometry. Plasma Sources Sci Technol 23:065025

28. Schulz C, Rolfes I (2014) The multipole resonance probe: progression and evaluation of a process compatible plasma sensor. IEEE Sensors J 14(10). Sensors Applications Symposium (SAS), IEEE

29. Schulz C, Styrnoll T, Awakowicz P, Rolfes I (2015) The planar multipole resonance probe: challenges and prospects of a planar plasma sensor. IEEE Trans Instrum Meas 64:14981187

Submit your manuscript to a SpringerOpen ${ }^{\odot}$ journal and benefit from:

- Convenient online submission

Rigorous peer review

- Open access: articles freely available online

- High visibility within the field

- Retaining the copyright to your article

Submit your next manuscript at $\boldsymbol{\text { springeropen.com }}$ 